\title{
BMJ Global Health Effectiveness of intermittent preventive treatment in pregnancy with sulphadoxine-pyrimethamine (IPTp-SP) in Ghana
}

David Kwame Dosoo (D) , ${ }^{1,2}$ Kezia Malm, ${ }^{3}$ Felix Boakye Oppong, ${ }^{2}$ Richard Gyasi, ${ }^{4}$ Abraham Oduro, ${ }^{5,6}$ John Williams, ${ }^{7}$ Dorcas Atibilla, ${ }^{2}$ Nana Yaw Peprah, ${ }^{3}$ Mieks Twumasi, ${ }^{2}$ Seth Owusu-Agyei, ${ }^{1,2,8}$ Brian Greenwood, ${ }^{1}$ Daniel Chandramohan, ${ }^{1}$ Kwaku Poku Asante ${ }^{1,2}$

To cite: Dosoo DK, Malm K, Oppong FB, et al. Effectiveness of intermittent preventive treatment in pregnancy with sulphadoxinepyrimethamine (IPTp-SP) in Ghana. BMJ Global Health 2021;6:e005877. doi:10.1136/ bmjgh-2021-005877

Handling editor Alberto L Garcia-Basteiro

- Additional supplemental material is published online only. To view, please visit the journal online (http://dx.doi.org/10. 1136/bmjgh-2021-005877).

Received 31 March 2021 Accepted 8 August 2021
Check for updates

(c) Author(s) (or their employer(s)) 2021. Re-use permitted under CC BY-NC. No commercial re-use. See rights and permissions. Published by BMJ.

For numbered affiliations see end of article.

Correspondence to Mr David Kwame Dosoo; david.dosoo@kintampo-hrc.org

\section{ABSTRACT}

Introduction Ghana adopted the revised WHO recommendation on intermittent preventive treatment in pregnancy using sulfadoxine-pyrimethamine (IPTp-SP) in 2012. This study has assessed the effectiveness and safety of this policy in Ghana.

Methods A total of 1926 pregnant women enrolled at antenatal care (ANC) clinics were assessed for birth outcomes at delivery, and placental histology results for malaria infection were obtained from 1642 participants. Association of reduced placental or peripheral malaria, anaemia and low birth weight (LBW) in women who received $\geq 4$ IPTp-SP doses compared with 3 or $\leq 2$ doses was determined by logistic regression analysis. Results Among the 1926 participants, 5.3\% (103), $19.2 \%$ (369), 33.2\% (640) and $42.3 \%$ (817) of women had received $\leq 1,2,3$ or $\geq 4$ doses, respectively. There was no difference in risk of active placental malaria (PM) infection in women who received 3 doses compared with $\geq 4$ doses (adjusted OR (aOR) 1.00, 95\% $\mathrm{Cl} 0.47$ to 2.14). The risk of overall PM infection was $1.63(95 \% \mathrm{Cl} 1.07$ to 2.48$)$ in 2 dose group and 1.06 (95\% $\mathrm{Cl} 0.72$ to 1.57$)$ in 3 dose group compared with $\geq 4$ dose group. The risk of LBW was $1.55(95 \% \mathrm{Cl} 0.97$ to 2.47$)$ and $1.06(95 \% \mathrm{Cl} 0.68$ to 1.65 ) for 2 and 3 dose groups, respectively, compared with the $\geq 4$ dose group. Jaundice in babies was present in $0.16 \%$, and $0 \%$ for women who received $\geq 4$ doses of SP. Conclusion There was no difference in the risk of PM, LBW or maternal anaemia among women receiving 3 doses compared with $\geq 4$ doses. Receiving $\geq 3$ IPTp-SP doses during pregnancy was associated with a lower risk of overall PM infection compared with 2 doses. As there are no safety concerns, monthly administration of IPTp-SP offers a more practical opportunity for pregnant women to receive $\geq 3$ doses during pregnancy.

\section{INTRODUCTION}

Malaria during pregnancy is a major public health problem that can lead to unfavourable pregnancy and birth outcomes such as abortion, preterm delivery, stillbirth, maternal

\section{Key questions}

What is already known?

- Malaria in pregnancy can lead to unfavourable pregnancy and birth outcomes such as abortion, preterm delivery, stillbirth, maternal anaemia and low birth weight (LBW).

- Intermittent preventive treatment during pregnancy using 3 or more doses of sulfadoxine-pyrimethamine (SP) has previously been shown to be more effective than 2 or fewer doses in controlling malaria in pregnancy.

- The National Malaria Control Programme of Ghana has transitioned from a 3-dose intermittent preventive treatment in pregnancy using SP (IPTp-SP) policy to the revised WHO policy, which recommends monthly administration of SP to pregnant women, starting early in the second trimester.

What are the new findings?

- Placental malaria was detected in $16.1 \%, 16.8 \%$, $10.4 \%$ and $9.7 \%$ of women who received $\leq 1,2,3$ or $\geq 4$ IPTp-SP doses, respectively.

- Risk of placental malaria, LBW and anaemia were not different in women who received 3 doses compared with $\geq 4$ doses.

- Incidence of jaundice in babies was present in $0 \%$, $0.28 \%, 0.32 \%$ and $0 \%$ for women who received $\leq 1$, 2,3 or $\geq 4$ doses of SP, respectively.

What do the new findings imply?

- There is no added benefit to receiving more than 3 doses of IPTp-SP. However, a monthly dosing schedule would facilitate women receiving at least 3 doses of IPTp-SP recommended by the WHO.

anaemia and low birth weight $(\mathrm{LBW}){ }^{1-3}$ In 2018, an estimated 11 million of 38 million (29\%) pregnancies were exposed to malaria in sub-Saharan Africa with $35 \%$ of pregnancies in West Africa exposed to malaria during the same period. ${ }^{4}$ 
To prevent malaria during pregnancy, and the unfavourable maternal and foetal outcomes associated with Plasmodium falciparum infections in areas of moderate to high malaria transmission, the WHO previously recommended the use of long-lasting insecticidetreated bednet (ITN), case management through prompt diagnosis and treatment as well as intermittent preventive treatment in pregnancy (IPTp) with at least 2 full treatment doses of sulfadoxine-pyrimethamine (SP) given after quickening in the second and third trimesters and before 36 weeks of gestation at intervals not less than 1 month. ${ }^{5}$ Effective IPTp, given at predetermined intervals during pregnancy, clears maternal parasitaemia and prevent subsequent reinfections. ${ }^{67}$ Ghana adopted this IPTp-SP policy in 2003, recommending that three tablets of $\mathrm{SP}$ should be given orally three times during pregnancy. ${ }^{8}$

The duration of the malaria prophylactic effect of SP has been reduced by the emergence of resistance to SP, for which reason it has been proposed that more frequent dosing may be needed. ${ }^{79}$ This supposition was supported by a meta-analysis of seven studies, which compared the efficacy of 2 doses to $\geq 3$ doses of IPTp-SP ${ }^{9}$ ) and showed the latter to be more efficacious. WHO policy on IPTp-SP was consequently revised and now recommends starting IPTp as early as possible in the second trimester, with administration at each scheduled ANC visit until the time of delivery, provided that the doses are given at least 1 month apart. Ghana adopted this revised policy in 2012. ${ }^{10}$ As a consequence of this change in policy, it has become possible for women to receive as many as six doses of SP during pregnancy, but the efficacy and safety of $>3$ doses of IPTp-SP have not been rigorously investigated. This study has, therefore, been undertaken to evaluate the impact of additional SP doses on PM, other malaria indicators, birth outcomes and safety, among pregnant Ghanaian women and their new borns.

\section{METHODS \\ Study areas}

This study was conducted in three different ecological zones of Ghana. The Dodowa area in the Greater Accra Region is in the forest-coastal savannah zone; Kintampo area in the Bono East Region is in the transition forest-savannah zone; Navrongo, in the Upper East Region, is in the Guinea savannah zone. Malaria transmission is low and perennial in the forest-coastal savannah zone, ${ }^{11}$ high and perennial in the transitional forest savannah zone ${ }^{12}$ and high but seasonal in the guinea savannah zone. ${ }^{13}$ The study was carried out between March 2017 and September 2019.

\section{Study design}

This was a prospective, observational, cohort study of pregnant women who attended ANC in the three study

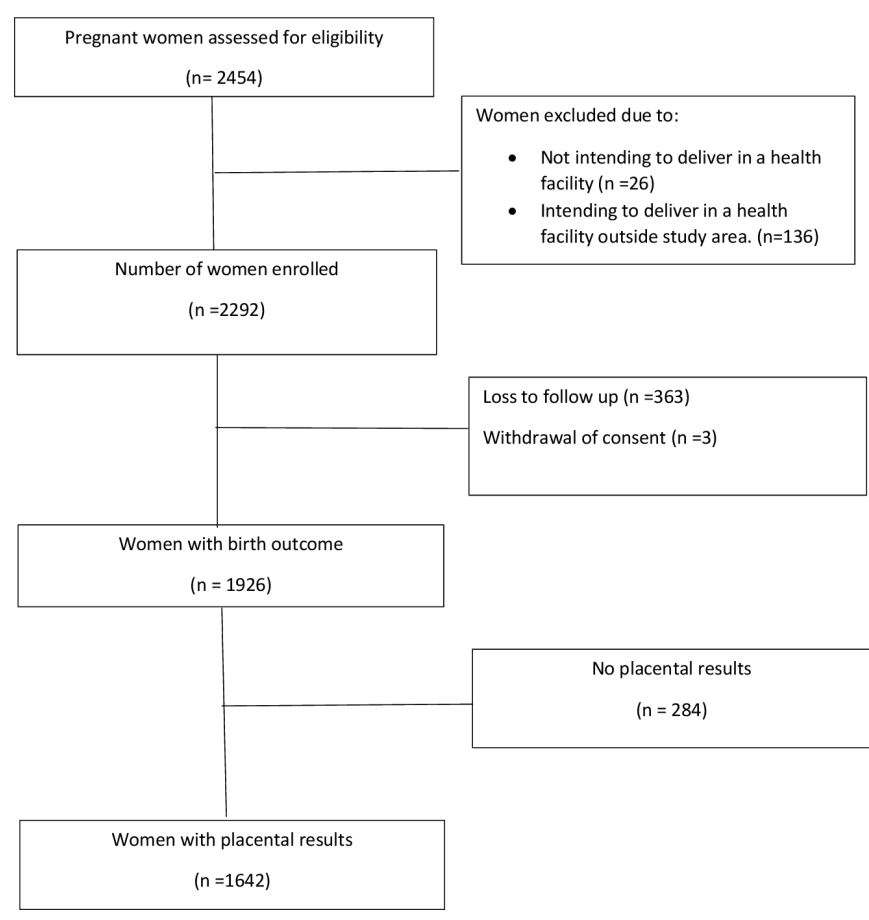

Figure 1 Flowchart of study.

sites. The study evaluated the effectiveness and safety of four or more doses of IPTp-SP on malaria in pregnancy and its consequences. The primary endpoint was active PM infection and secondary endpoints were overall PM infection, maternal anaemia, LBW, maternal peripheral blood malaria parasitaemia, placental blood and cord blood parasitaemia and maternal and fetal outcomes at delivery. Women of any gravidity attending an ANC who were aged 18 years and above with an intention to deliver in a health facility were eligible to join the study. Women with serious illness requiring hospitalisation, known HIV infection or known full deficiency of glucose-6-phosphate dehydrogenase, were excluded.

\section{Sample size}

The prevalence of active PM infection within the middle belt of Ghana in 2013 was $7.4 \% .{ }^{14}$ Although this prevalence could have changed, it represents the best estimate for active PM in this part of the country where the majority of the women was enrolled. To detect a reduction in active P. falciparum PM infection from $7.4 \%$ to $3.4 \%$, with a 0.05 level of significance and $80 \%$ power, a sample of at least 502 women who receive $\geq 4$ doses of IPTp-SP compared with an equal number of those who received 3 doses of IPTp-SP was required. Assuming a 10\% loss to follow-up during the course of pregnancy, an additional 50 women were needed for each group making a total of 1104women required for the study (Open Epi Software). Annual data from the study areas in 2015 and 2016 showed approximately $50 \%$ of pregnant women received 3 doses of IPTp-SP. There was, therefore, the need to increase the sample size by a minimum of $50 \%$ (ie, 552) to be able to achieve at least 502 participants at delivery for each group. 
Table 1 Characteristic of study participants at enrolment by doses of SP used by the time of delivery

\begin{tabular}{|c|c|c|c|c|c|c|c|c|c|c|}
\hline \multirow[b]{2}{*}{ Characteristic } & \multicolumn{2}{|c|}{$\begin{array}{l}\text { Overall } \\
(\mathrm{N}=1926)\end{array}$} & \multicolumn{2}{|c|}{$\begin{array}{l}\leq 1 \text { dose } \\
(\mathrm{N}=103)\end{array}$} & \multicolumn{2}{|c|}{$\begin{array}{l}2 \text { doses } \\
(\mathrm{N}=369)\end{array}$} & \multicolumn{2}{|c|}{$\begin{array}{l}3 \text { doses } \\
(\mathrm{N}=640)\end{array}$} & \multicolumn{2}{|c|}{$\begin{array}{l}\geq 4 \text { doses } \\
(\mathrm{N}=814)\end{array}$} \\
\hline & $\mathbf{N}$ & $\%$ & $\mathbf{N}$ & $\%$ & $\mathbf{N}$ & $\%$ & $\mathbf{N}$ & $\%$ & $\mathbf{N}$ & $\%$ \\
\hline \multicolumn{11}{|l|}{ Study site } \\
\hline Kintampo & 1128 & 58.6 & 40 & 38.8 & 202 & 54.7 & 365 & 57.0 & 521 & 64.0 \\
\hline Dodowa & 404 & 21.0 & 34 & 33.0 & 105 & 28.5 & 159 & 24.8 & 106 & 13.0 \\
\hline Navrongo & 394 & 20.4 & 29 & 28.2 & 62 & 16.8 & 116 & 18.1 & 187 & 23.0 \\
\hline \multicolumn{11}{|l|}{ Age } \\
\hline Mean (SD) & \multicolumn{2}{|c|}{$27.69(6.01)$} & \multicolumn{2}{|c|}{$27.26(6.62)$} & \multicolumn{2}{|c|}{$27.25(6.28)$} & \multicolumn{2}{|c|}{$27.90(6.02)$} & \multicolumn{2}{|c|}{$27.78(5.78)$} \\
\hline \multicolumn{11}{|l|}{ Age group } \\
\hline 18-24 years & 647 & 33.6 & 40 & 38.8 & 141 & 38.2 & 202 & 31.6 & 264 & 32.4 \\
\hline 25-34 years & 995 & 51.7 & 45 & 43.7 & 177 & 48.0 & 345 & 53.9 & 428 & 52.6 \\
\hline$\geq 35$ years & 284 & 14.7 & 18 & 17.5 & 51 & 13.8 & 93 & 14.5 & 122 & 15.0 \\
\hline \multicolumn{11}{|l|}{ Level of education } \\
\hline No education & 425 & 22.1 & 25 & 24.3 & 103 & 27.9 & 141 & 22.0 & 156 & 19.2 \\
\hline Primary & 306 & 15.9 & 16 & 15.5 & 63 & 17.1 & 100 & 15.6 & 127 & 15.6 \\
\hline Junior high & 695 & 36.1 & 38 & 37.0 & 133 & 36.0 & 242 & 37.8 & 281 & 34.5 \\
\hline Secondary or higher & 500 & 25.9 & 24 & 23.3 & 70 & 19.0 & 157 & 24.5 & 250 & 30.7 \\
\hline \multicolumn{11}{|l|}{ Marital status } \\
\hline Married/married before & 1450 & 75.3 & 75 & 72.8 & 242 & 65.6 & 490 & 76.6 & 643 & 79.0 \\
\hline Living together with man, unmarried & 263 & 13.7 & 17 & 16.5 & 72 & 19.5 & 91 & 14.2 & 83 & 10.2 \\
\hline Single, unmarried & 213 & 11.0 & 11 & 10.7 & 55 & 14.9 & 59 & 9.2 & 88 & 10.8 \\
\hline \multicolumn{11}{|l|}{ Religious background } \\
\hline Christian & 1475 & 76.6 & 83 & 80.6 & 267 & 72.4 & 485 & 75.8 & 640 & 78.6 \\
\hline Muslim & 389 & 20.2 & 17 & 16.5 & 85 & 23.0 & 132 & 20.6 & 155 & 19.0 \\
\hline Traditionalist & 11 & 0.6 & 0 & 0.0 & 5 & 1.4 & 4 & 0.6 & 2 & 0.3 \\
\hline Other & 51 & 2.6 & 3 & 2.9 & 12 & 3.3 & 19 & 3.0 & 17 & 2.1 \\
\hline \multicolumn{11}{|l|}{ Household SES } \\
\hline Poorest & 386 & 20.0 & 27 & 26.2 & 96 & 26.0 & 129 & 20.2 & 134 & 16.5 \\
\hline More poor & 385 & 20.0 & 23 & 22.3 & 62 & 16.8 & 124 & 19.4 & 176 & 21.6 \\
\hline Poor & 385 & 20.0 & 20 & 19.4 & 78 & 21.1 & 113 & 17.7 & 174 & 21.4 \\
\hline Less poor & 385 & 20.0 & 19 & 18.5 & 73 & 19.8 & 138 & 21.6 & 155 & 19.0 \\
\hline Least poor & 385 & 20.0 & 14 & 13.6 & 60 & 16.3 & 136 & 21.3 & 175 & 21.5 \\
\hline \multicolumn{11}{|l|}{ Gravidity } \\
\hline Primigravidae & 436 & 22.6 & 29 & 28.2 & 89 & 24.1 & 133 & 20.8 & 184 & 22.6 \\
\hline Secundigravidae & 474 & 24.6 & 22 & 21.4 & 92 & 24.9 & 163 & 25.5 & 197 & 24.2 \\
\hline Multigravidae & 1016 & 52.8 & 52 & 50.5 & 188 & 51.0 & 344 & 53.8 & 433 & 53.2 \\
\hline \multicolumn{11}{|l|}{ ITN use } \\
\hline Yes & 1242 & 64.5 & 70 & 68.0 & 232 & 62.9 & 393 & 61.4 & 547 & 67.2 \\
\hline No & 684 & 35.5 & 33 & 32.0 & 137 & 37.1 & 247 & 38.6 & 267 & 32.8 \\
\hline
\end{tabular}

ITN, insecticide-treated bednet; SES, socioeconomic status; SP, sulfadoxine-pyrimethamine.

\section{Enrolment procedures}

Fieldworkers, laboratory personnel and midwives were trained on study procedures prior to commencement of the study. Informed consent was obtained from all eligible pregnant women and a structured questionnaire was completed as previously described. ${ }^{15}$ Gestational age was determined by midwives at the first ANC visit using the symphysis-fundal height measurement method ${ }^{16}$ and subsequently using the ultrasound technique. IPTp-SP was administered by nurses at ANC clinics, following the WHO-revised policy guidelines and SP administration was recorded in the participant's antenatal book at each visit.

\section{Delivery}

At delivery, cord blood ( $5 \mathrm{~mL})$ was collected, after clamping the umbilical cord, into a $\mathrm{K}_{2}$-EDTA tube 


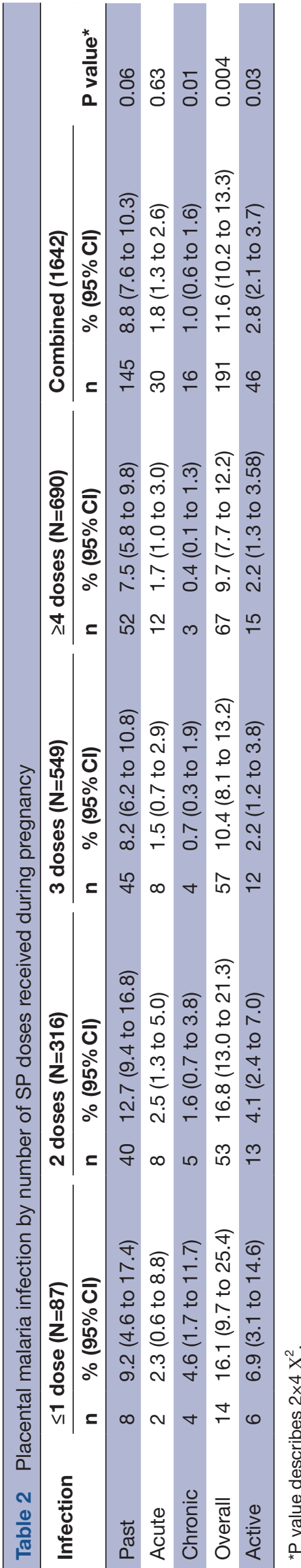

(Becton Dickinson, England) for determination of haemoglobin $(\mathrm{Hb})$ concentration and malaria parasitaemia by microscopy. A placental biopsy (approximately $2.5 \mathrm{~cm}^{3}$ ) was taken from the maternal side of the placenta within 1 hour of placental expulsion and placed in a prelabelled bottle containing $50 \mathrm{~mL}$ of $10 \%$ neutral buffered formalin. Incisions were also made on the maternal side of the placenta and $0.5 \mathrm{~mL}$ of placental blood collected, using a Pasteur pipette, into an EDTA Microtainer tube (Becton Dickinson, England). A capillary blood sample $(0.2 \mathrm{~mL})$ was collected from the mother prior to delivery for malaria microscopy. All biological samples were transported in a cold box to the nearest clinical laboratory (Kintampo Health Research Centre, Navrongo Health Research Centre or Shai-Osudoku District Hospital) for processing. New-borns were weighed on a calibrated digital scale (Jactermac, Germany) to the nearest $0.1 \mathrm{~kg}$ within 24 hours of birth. Screening for congenital abnormalities was performed by 2 midwives using clinical examination.

\section{Laboratory procedures}

$\mathrm{Hb}$ estimation

$\mathrm{Hb}$ concentration was estimated using a validated $\mathrm{ABX}$ Pentra 60C+Haematology analyser (Horiba-ABX, Montpellier, France) or a HemoCue 200+haemoglobinometer (HemoCue, Angelholm, Sweden) according to manufacturers' operating instructions. Quality controls were performed to ensure quality of the results generated.

\section{Malaria parasitaemia determination}

Thick and thin malaria blood smears were prepared from peripheral, placental and cord blood and stained using standard procedures, as described previously. ${ }^{17} 18$ Briefly, thick and thin blood smears were prepared on the same slide, air-dried and the thin smear fixed with absolute methanol. Both smears were stained with $10 \%$ Giemsa for $10 \mathrm{~min}$ and air-dried. Parasites were detected and counted in the thick smear when $<100$ parasites were seen in a high power field, otherwise, counting was done using the thin smear. Parasite density was calculated using absolute or, if white blood cell or red cell count was not available, using an assumed leucocyte $\left(8000 / \mathrm{cm}^{3}\right)$ or erythrocyte $\left(5000000 / \mathrm{cm}^{3}\right)$ count. A slide was declared as negative when no parasites were seen after examining at least 200 high power fields. Slides were examined by two certified independent microscopists and by a third in cases of discordance between the two initial readers. Differences were resolved by taking the result of the third reader and that of the earlier reader who agreed with him/her and in case of positive slides the average parasite density was used.

\section{PM infection determination}

Placental biopsies, placed in 10\% neutral buffered formalin, were stored in an air-conditioned room and transported to the Pathology Department of the School of Biomedical and Allied Health Sciences of the University 
Table 3 Crude and adjusted ORs of 2 doses of IPTp-SP versus 3 or more doses

\begin{tabular}{|c|c|c|c|c|c|c|}
\hline \multirow[b]{2}{*}{ Outcome variable } & \multicolumn{3}{|c|}{ Unadjusted analysis } & \multicolumn{3}{|c|}{ Adjusted analysis* } \\
\hline & cOR & $95 \% \mathrm{Cl}$ & $P$ value & aOR & $95 \% \mathrm{Cl}$ & P value \\
\hline \multicolumn{7}{|c|}{ Maternal parasitemia } \\
\hline 2 doses & 2.08 & 1.14 to 3.81 & 0.02 & 1.56 & 0.84 to 2.93 & 0.16 \\
\hline$\geq 3$ doses & 1 & & & 1 & & \\
\hline \multicolumn{7}{|c|}{ Active placental infection } \\
\hline 2 doses & 1.96 & 1.01 to 3.81 & 0.05 & 1.45 & 0.73 to 2.89 & 0.29 \\
\hline$\geq 3$ doses & 1 & & & 1 & & \\
\hline \multicolumn{7}{|c|}{ Overall placental infection } \\
\hline 2 doses & 1.82 & 1.29 to 2.57 & 0.001 & 1.58 & 1.08 to 2.29 & 0.02 \\
\hline$\geq 3$ doses & 1 & & & 1 & & \\
\hline \multicolumn{7}{|l|}{ Maternal anaemia } \\
\hline 2 doses & 1.32 & 1.03 to 1.70 & 0.03 & 1.19 & 0.92 to 1.54 & 0.18 \\
\hline$\geq 3$ doses & 1 & & & 1 & & \\
\hline \multicolumn{7}{|l|}{ Low birth weight } \\
\hline 2 doses & 1.72 & 1.14 to 2.58 & 0.01 & 1.52 & 0.99 to 2.32 & 0.05 \\
\hline$\geq 3$ doses & 1 & & & 1 & & \\
\hline
\end{tabular}

${ }^{*}$ Adjusted for age, education, household SES, marital status, gravidity, ITN use, malaria treatment in past 2 weeks and malaria treatment during pregnancy.

aOR, adjusted OR; IPTp-SP, intermittent preventive treatment in pregnancy using sulfadoxine-pyrimethamine; ITN, insecticide-treated bednet; SES, socioeconomic status.

of Ghana and processed as described previously. ${ }^{19}$ Briefly, biopsies processed and embedded in paraffin wax blocks were sectioned and two slides were stained with $\mathrm{H} \& \mathrm{E}$ and Giemsa stains and examined microscopically by a pathologist for the presence of malaria parasites and/ or pigment. Results were reported as no infection, acute infection, chronic infection or past infection, based on the Ishmail modification of Bulmer's classification. Active PM infection was defined as the presence of either acute or chronic placental infection. ${ }^{20}$ As part of quality control, $10 \%$ of slides were examined by an independent pathologist and results compared with those of the first reader (online supplemental table 1 ).

\section{Outcome definitions}

Active PM infection was defined as the presence of malaria parasites in the placenta with or without the presence of pigment, while overall placental infection was defined as the presence of parasite and/or pigment. ${ }^{20}{ }^{21}$ Maternal anaemia was defined as a $\mathrm{Hb}$ concentration $<110 \mathrm{~g} / \mathrm{L}{ }^{22}$ LBW was defined as a singleton baby weighing $<2.5 \mathrm{~kg}$ measured within 24 hours after birth; stillbirth as a delivery of a non-living fetus $\geq 28$ weeks gestation; preterm delivery as a newborn with a gestational age $<37$ weeks at delivery. Neonatal anaemia was defined as a cord blood $\mathrm{Hb}$ concentration $<125 \mathrm{~g} / \mathrm{L}^{23}$

\section{Data management and statistical analysis}

All data were recorded on structured questionnaires, checked for completeness, double-entered and verified using a database designed with C-Sharp application as front end and SQL-Server as back end. Statistical analysis was carried out using Stata V.15 (StataCorp, College Station, USA).

Uptake of IPTp-SP was grouped into $\leq 1,2,3$ and $\geq 4$ doses. $\chi^{2}$ tests were used to explore the association between the characteristics of study participants at enrolment and the number of doses of IPTp-SP received. The association between the number of doses of IPTp-SP received and $\mathrm{PM}$ infection (past, acute, chronic, overall and active) and birth outcomes (preterm delivery, LBW, mode of delivery, delivery complication, congenital abnormalities, neonatal jaundice, neonatal anaemia) were assessed using $\chi^{2}$ or Fisher's exact tests, where applicable.

Logistic regression was used to assess the association between the number of doses of IPTp-SP received and active PM infection, overall PM infection, maternal peripheral malaria parasitaemia, maternal anaemia at delivery and LBW. In each regression model, together with number of doses of IPTP-SP received, univariate analyses with age, education, household wealth, marital status, gravidity, ITN use, malaria treatment in the past 2 weeks and malaria treatment during pregnancy were performed first. Variables found in the univariates analyses to be significant at 0.15 level of significance were included in the multivariate analyses. In the multivariate analyses, variables with a $\mathrm{p}$ value $<0.05$ were considered to be statistically significant. Active PM was found in only $2.8 \%(46 / 1642)$ of the participants with histologically examined placental biopsies, while $2.9 \%$ (54/1886) of the participants had peripheral blood asexual $P$. falciparum 


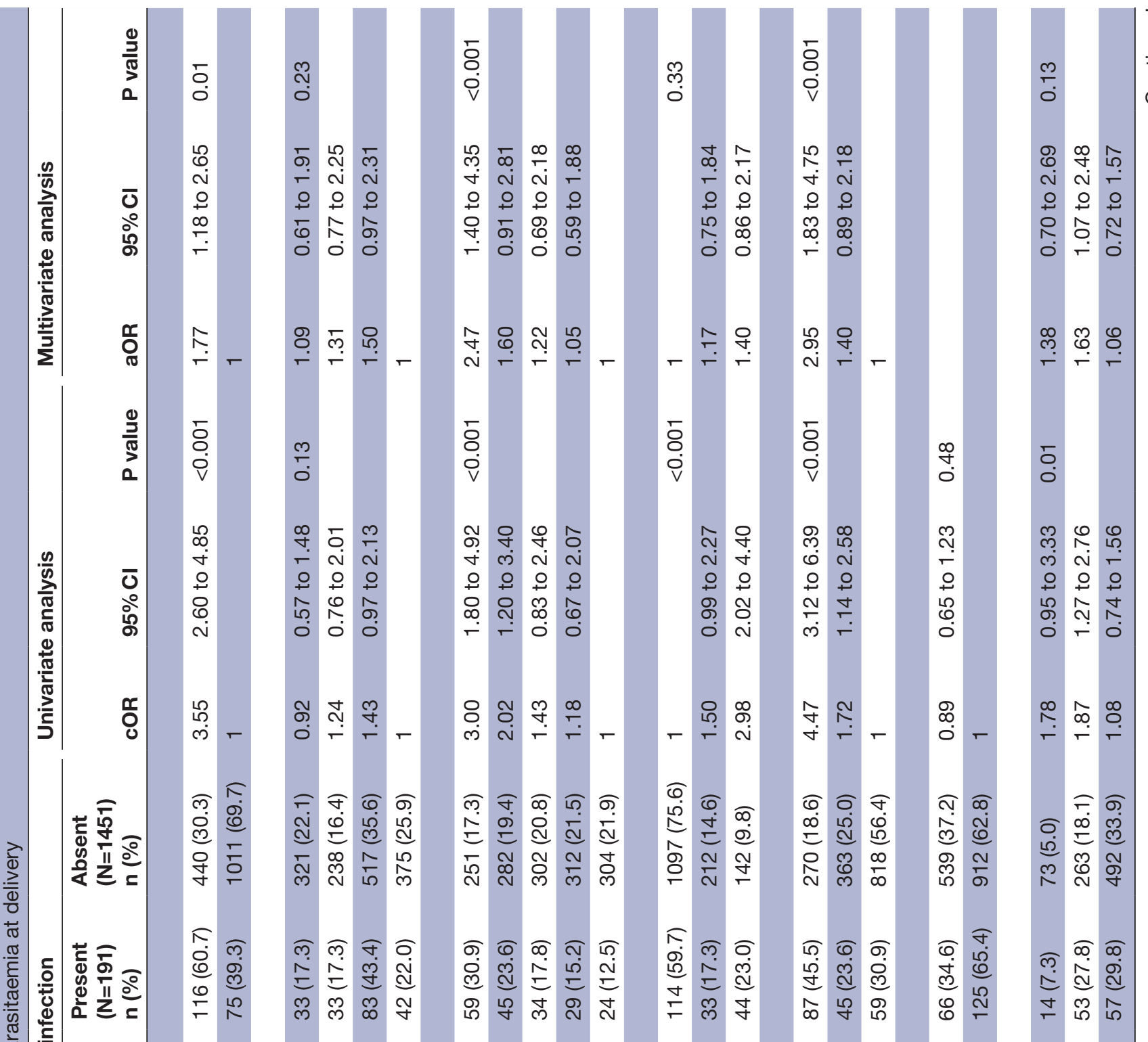

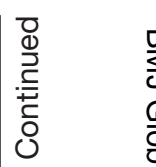

ㄴํำํํㅇำ

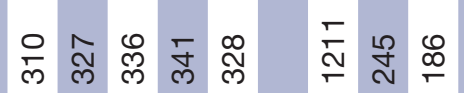

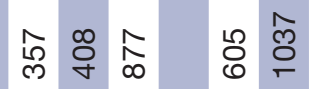

๙ $\frac{0}{m} \frac{9}{5}$

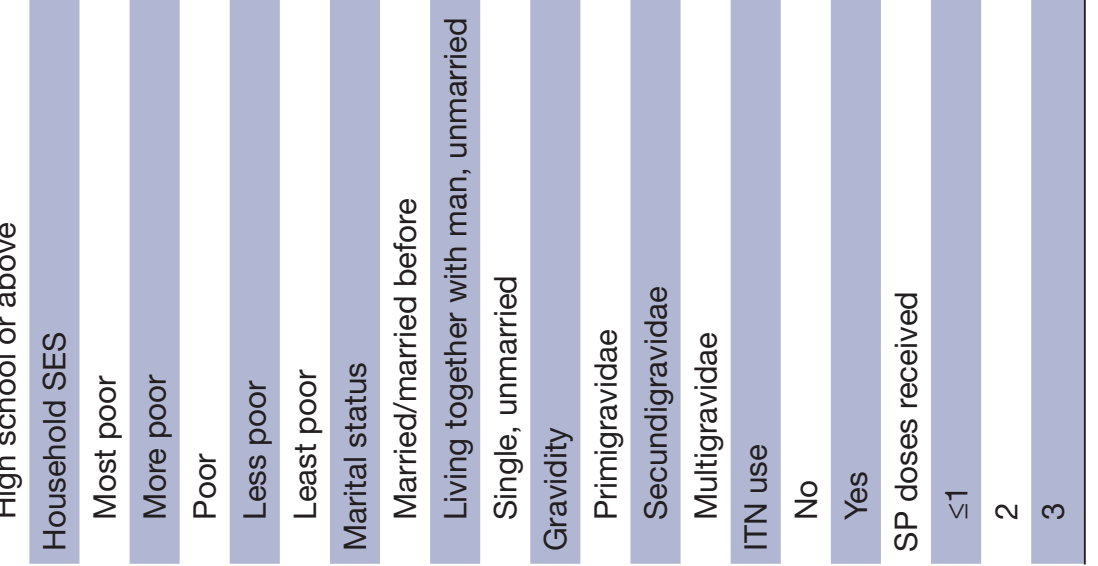




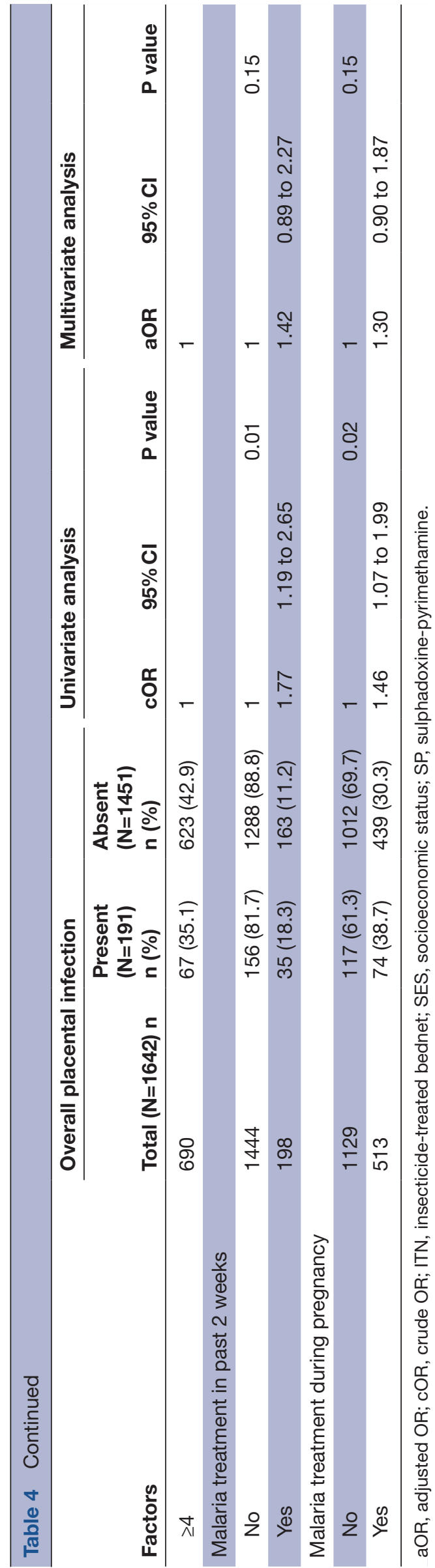

infection at delivery. Consequently, in accounting for the bias associated with using logistic regression for rare events, the penalised likelihood approach, which reduces the small-sample bias in maximum likelihood estimation was used. ${ }^{24}$

\section{Patient and public involvement}

Patients or the public were not involved in the design, or conduct, or reporting, or dissemination plans of our research.

\section{RESULTS}

\section{Characteristics of study participants}

A total of 2454 pregnant women were screened for eligibility and $93.5 \%$ (2292/2454) were enrolled. Delivery outcomes were obtained for $84.0 \%(1926 / 2292)$ and placental results were available for $85.3 \%$ (1642/1926) (figure 1). There was no difference in characteristics between participants with or without placental results (online supplemental table 2). At enrolment, 52.2\% (1006/1926) and $47.8 \%(920 / 1926)$ of participants had gestational age determined using ultrasound and fundal height, respectively.

Among the participants with birth outcomes, those who had taken $0,1,2,3$ or $\geq 4$ doses by the time of delivery were $0.7 \%$ (14), $4.6 \%$ (89), $19.2 \%$ (369), 33.2\% (640) and $42.3 \%$ (814), respectively. Majority of the study population, $58.6 \%(1128 / 1926)$ came from Kintampo area and $51.2 \%$ (978/1926) were aged between 25 and 34 years. Primigravidae and secundigravidae formed $22.6 \%$ $(435 / 1926)$ and $24.7 \%(475 / 1926)$ of the study population, respectively, and $64.5 \%(1242 / 1926)$ had used an ITN the previous night (table 1$)$. Only 29\% (562/1926) of the women reported treatment for malaria during pregnancy while $11.6 \%(223 / 1926)$ reported receiving antimalarial treatment within 14 days prior to delivery. Characteristics of study participants by study area are presented in online supplemental table 3 .

\section{Impact of IPTp-SP on PM infection}

The prevalence of overall PM among the 1642 placental biopsies examined histologically was $11.6 \%$ (95\% CI $10.2 \%$ to $13.3 \%)$. This comprised $2.8 \%$ active infections (1.8\% acute and $1.0 \%$ chronic) and $8.8 \%$ past infections.

The prevalence of active placental infection was highest in women who received $\leq 1$ dose $(6.9 \%)$, followed by those who received 2 doses $(4.1 \%)$ but the same, $2.2 \%$ in each case, among those who received 3 or $\geq 4$ doses of IPTp-SP (table 2). In the univariate analysis, age, household socioeconomic status (SES), marital status, gravidity and the number of SP doses received during pregnancy were associated with active placental infection. In the multivariate analysis, there was no difference in the risk of active $\mathrm{PM}$ infection among women who received 3 or $\geq 4$ doses of IPTp-SP (adjusted OR (aOR) 1.00, 95\% CI 0.47 to 2.15 ) nor between those who received 2 versus $\geq 4$ doses (aOR 1.48, 95\% CI 0.69 to 3.18) (online supplemental table 4). There was also no difference in risk of active PM 
infection in women who received 2 doses compared with those who received $\geq 3$ doses IPTp-SP (aOR 1.45, 95\% CI 0.73 to 2.89 ) (table 3 ).

Lower age, lower household SES, being single, primigravid or secundigravid and receiving malaria treatment during pregnancy were associated with overall PM infection in the univariate analysis. In the multivariate analysis, risk of overall PM infection was high in women aged $<25$ years compared with those aged $\geq 25$ years (aOR $1.77,95 \%$ CI 1.18 to 2.65); the most poor household SES compared with the least poor (aOR 2.47, 95\% CI 1.40 to 4.35 ); primigravidae compared with multigravidae (aOR $2.95,95 \%$ CI 1.83 to 4.75 ) and those who received $\leq 2$ doses IPTp-SP compared with those who received $\geq 4$ doses (aOR 1.63, 95\% CI 1.07 to 2.48) (table 4). The risk of overall PM infection was also higher in women who received 2 doses compared with those who received $\geq 3$ doses IPTp-SP (aOR 1.58, 95\% CI 1.08 to 2.29) (table 3).

The overall prevalence of malaria parasitaemia in placental blood was $1.8 \%$ (25/1387). The prevalence of placental blood malaria was highest in women who received $\leq 1$ dose $(2.9 \% ; 2 / 69)$ and lowest in those who received $\geq 4$ doses $(1.4 \% ; 8 / 587)$. The number of IPTp-SP doses received during pregnancy had no impact on the prevalence of placental blood malaria.

\section{The impact of IPTp-SP on maternal peripheral and cord blood malaria parasitaemia at delivery}

Overall, the prevalence of peripheral blood asexual $P$. falciparum infection at delivery among study women was $2.9 \%(54 / 1886)$. Among women who received $\leq 1$, 2,3 and $\geq 4$ doses of IPTp-SP, the prevalence was $6.2 \%$ $(6 / 97), 4.5 \%(16 / 357), 2.6 \%(16 / 627)$ and $2.0 \%$ $(16 / 805)$, respectively, but differences between groups were not statistically significant. In the univariate analysis, age, household SES, number of SP doses received and malaria treatment during pregnancy were associated with a higher risk of maternal peripheral malaria parasitaemia at delivery. In the multivariate analysis, there was no difference in risk of peripheral malaria parasitaemia in women who received 3 or $\geq 4$ doses of $\mathrm{SP}$ (aOR 1.29, $95 \%$ CI 0.64 to 2.60) nor 2 doses versus $\geq 4$ doses of SP (aOR 1.74, 95\% CI 0.85 to 3.56). Being in a household of lower SES remained strongly associated with a risk of peripheral malaria parasitaemia (table 5). The overall prevalence of cord blood $P$. falciparum parasitaemia was $0.4 \%$, and $0 \%(0 / 74), 0 \%(0 / 294), 0.8 \%(4 / 503)$ and $0.3 \%(2 / 643)$ among women who received $\leq 1,2,3$ and $\geq 4$ doses of SP, respectively. There was no difference by number of IPTp-SP doses received.

\section{Impact of IPTp-SP on maternal anaemia at delivery}

The overall mean $\mathrm{Hb}$ concentration (SD) for study women at delivery was $11.5(2.0) \mathrm{g} / \mathrm{dL}$. Overall, the prevalence of maternal anaemia $(\mathrm{Hb}<11.0 \mathrm{~g} / \mathrm{dL})$ was $34.2 \%$. The prevalence of anaemia by number of IPTp-SP doses received was $40.4 \%$ (40/90), 38.8\% (130/335) 35.5\% (206/581) and $30.0 \%(222 / 741)$ in women who received $\leq 1,2,3$ and $\geq 4$ doses of IPTp-SP respectively.

In a univariate analysis, low household SES, being unmarried, receiving fewer IPTp-SP doses during pregnancy and having been treated for malaria (apart from SP) were associated with a higher risk of maternal anaemia at delivery (online supplemental table 5). In the multivariate analysis, the risk of anaemia was higher in women from most poor household compared with those from least poor households (aOR 1.51, 95\% CI 1.09 to 2.06) and women who received 2 IPTp-SP doses during pregnancy compared with those who received $\geq 4$ doses (aOR 1.34, 95\% CI 1.02 to 1.77). There was no statistically significant difference in risk of anaemia among women who received 3 doses compared with those who received $\geq 4$ doses (aOR 1.24, 95\% CI 0.99 to 1.56 ) (online supplemental table 5) and also in women who received 2 doses compared with those who received $\geq 3$ doses (aOR 1.19, 0.92-1.54) (table 3).

\section{Impact of IPTp-SP on LBW and other birth outcomes}

Overall, $0.5 \%(10 / 1926)$ and $0.9 \%(17 / 1926)$ of pregnancies resulted in a miscarriage or stillbirth, respectively. The mean gestational age at delivery overall was 38.4 (SD 2.5 ) weeks and increased significantly with an increase in the number of IPTp-SP doses received (table 6).

Mean birth weight for all live singleton deliveries was 3.07 (SD 0.48) kg, and $2.84 \mathrm{~kg}, 2.99 \mathrm{~kg}, 3.09 \mathrm{~kg}$ and $3.11 \mathrm{~kg}$ in babies of women who received $\leq 1,2,3$, or $\geq 4 \mathrm{SP}$ doses, respectively. The overall prevalence of LBW was $7.5 \%(140 / 1856)$ and $17.9 \%$ (17/95), 10.2\% (36/354), $6.5 \%(40 / 615)$ and $5.9 \%(47 / 792)$ in babies of women who received $\leq 1,2,3$ or $\geq 4$ SP doses, respectively. Factors strongly associated with LBW in the univariate analysis were household SES, gravidity and the number of IPTp-SP doses received. In the multivariate analysis, the risk of LBW was higher in babies born to women of most poor households compared with those from least poor households (aOR 4.26, 95\% CI 2.23 to 8.04), There was no difference in risk of LBW between babies of women who received 3 doses of $\mathrm{SP}$ and those who received $\geq 4$ doses (aOR 1.06, 95\% CI 0.68 to 1.65 ) nor between those who received 2 doses and those who received $\geq 4$ doses (table 7). The risk of LBW in babies born to mothers who received 2 doses of IPTp-SP did not differ significantly compared with those who received $\geq 3$ doses (aOR 1.52, 0.99 to 2.32 ) (table 3 ).

\section{Impact of IPTp-SP doses on the safety of the baby}

A low prevalence of jaundice, defined as vellowish coloration of the baby's eyes $(0.16 \%, 9 / 1899)$, was observed in the babies overall, with none in those born to women who received $\geq 4$ doses of SP during pregnancy (table 6 ). The mean haemoglobin concentration in study babies overall was $14.53 \mathrm{~g} / \mathrm{dL}$ and highest $(14.62 \mathrm{~g} / \mathrm{dL})$ in the babies born to women who received $\geq 4$ doses of SP during pregnancy, but with no significant differences between the groups. The overall prevalence of neonatal 


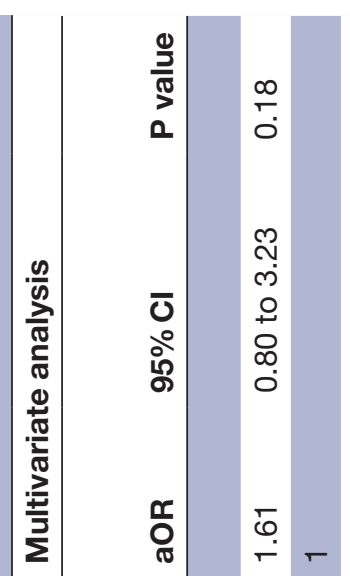

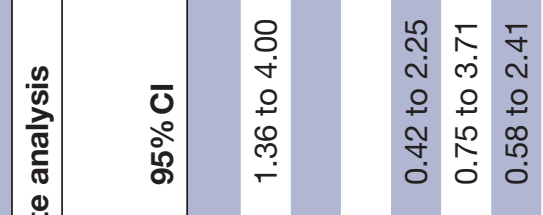

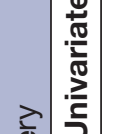

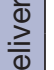

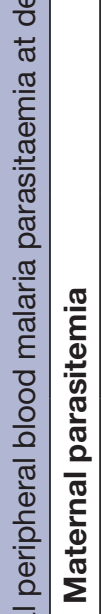

萬

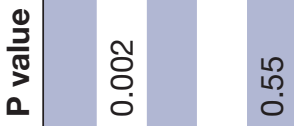

$\bar{\varnothing}$

员

ㅇㅇㅇㅇㅇㅇ

守

ర్ల

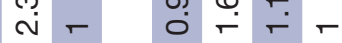

ब

궐

品嵒 苍

రั

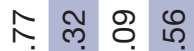

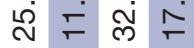

웅ㅇㅇㅇㅇ

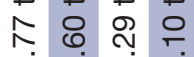

- O

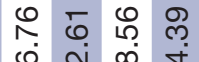

กิ ㅇํ

ণ্ণ

$\underset{\substack{\infty \\ 0}}{\substack{\infty \\ 0}}$

ำ ธก รั

ن่

우우

โ⿳⺈冂大

号

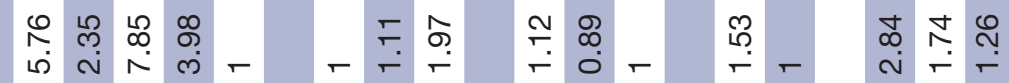

ষ্ণ

$\stackrel{\infty}{\circ}$

$\stackrel{\infty}{\circ}$

$\underset{0}{0}$

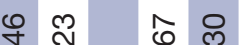

लं

웅

사유

우우

它

O

స.

กิ

t)

स

$\infty$ \&ं

우우

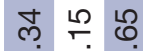

-

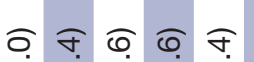

वृ จ्ปे

잉

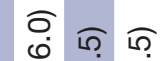

$\stackrel{5}{\circ} \stackrel{0}{0}$

寅离

สิ

철 这

过售㔯

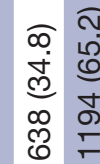

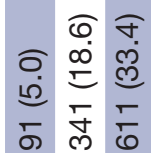

กิ $\widehat{x}=\widehat{x}$

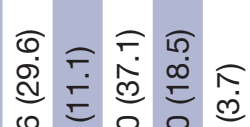

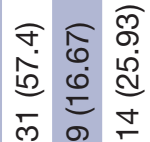

สิ สิ

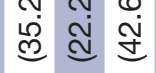

ल

过

ผ

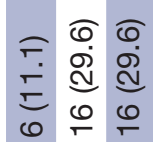

ธุ สุ

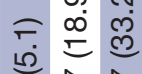

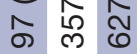

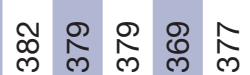

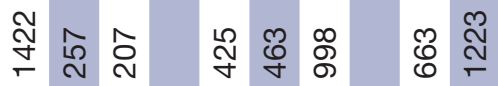

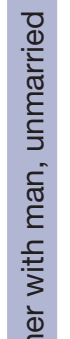

은 등

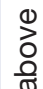

ํํำ

要

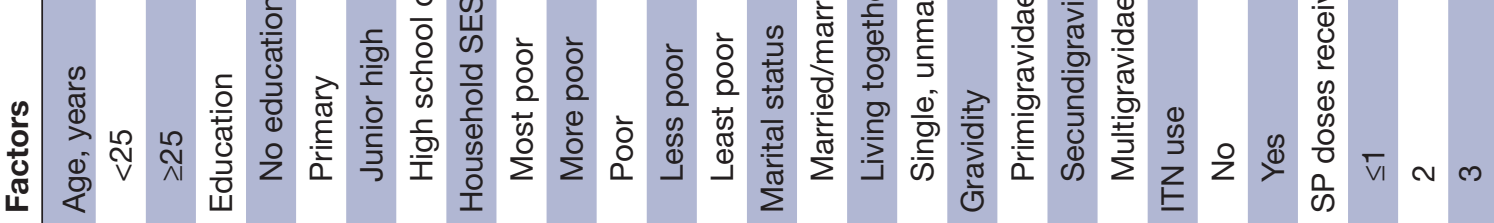

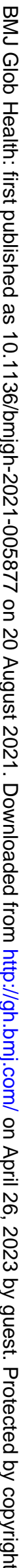




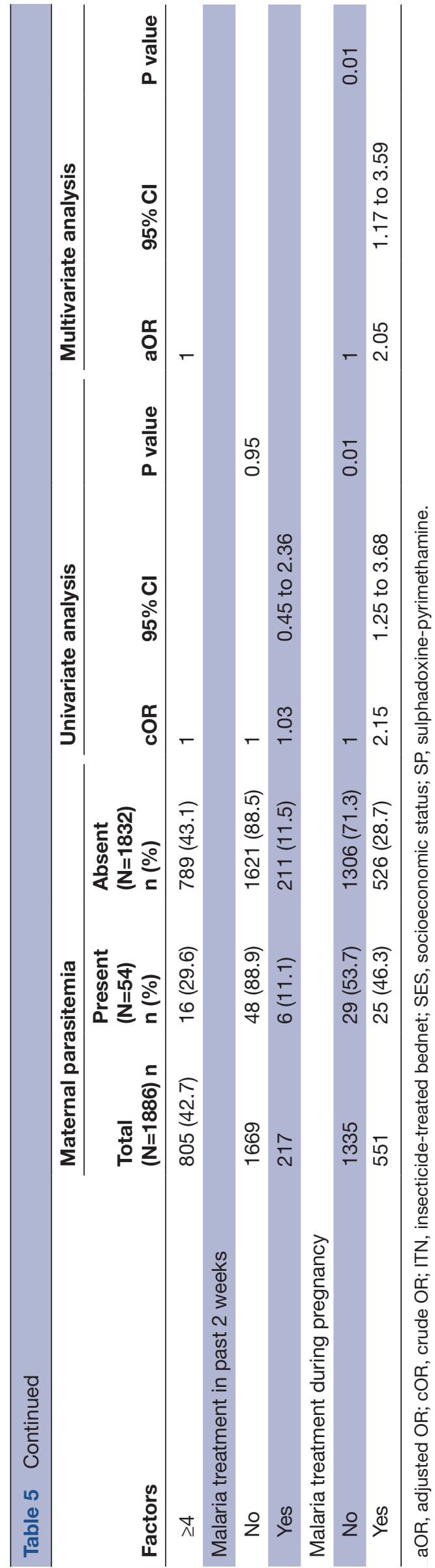

anaemia was $15.4 \%$ (266/1731); $21.1 \%$ (19/90), $16.5 \%$ (55/333), $16.6 \%(96 / 580)$ and $13.2 \%(96 / 728)$ in babies of mothers who received $\leq 1,2,3$ and $\geq 4$ SP doses, respectively (table 6). The overall prevalence of congenital abnormalities was $0.5 \%(9 / 1899)$ and there was no statistical difference in the incidence of congenital abnormalities by the number of IPTp-SP doses that the mother had received (table 6 ).

\section{DISCUSSION}

This observational, prospective cohort study demonstrated a low prevalence of both active and overall histologically confirmed PM infection in three ecological zones in the northern guinea-savannah, middle forestsavannah and southern forest-coastal-savannah zones of Ghana. Our finding of no statistically significant benefit of receiving $\geq 4$ doses IPTp-SP over the standard 3-dose in Ghana against PM (active or overall), peripheral maternal malaria, LBW or maternal anaemia is consistent with findings in Zambia. ${ }^{25} 26$

The nearly two-fold risk of overall PM infection in women who received 2 doses compared with those who received 3 or more doses in the present study is consistent with previous ones that reported 3 or more doses of IPTp-SP to be more effective in reducing PM than 2 doses. ${ }^{27}$ The prevalence of active PM found in this study was lower than in the northern and middle zones of Ghana in 2013 and 2015, respectively, prior to implementation of the WHO monthly IPTp-SP policy. ${ }^{1428}$ The prevalence of overall PM infection of $11.6 \%$ was lower than in the southern coastal $(35.9 \%)$ and middle forest-savannah $(37.5 \%)$ zones of Ghana, respectively, before introduction of the new policy. This marked reduction in the prevalence of PM infection could be attributed in part to high ITN use, use of other malaria prevention interventions as well as to a high uptake of IPTp-SP in the study cohort. A baseline study on the prevalence of malaria parasitaemia among pregnant women at first ANC visit in our study area in the middle belt of Ghana showed one-fifth of the women carried malaria parasites. ${ }^{15}$ The low malaria parasite prevalence reported in this study can further be reduced if IPTp-SP uptake continues to increase over time.

Contrary to findings in previous studies in Ghana ${ }^{29} 30$ and elsewhere, ${ }^{29}$ this study showed no difference in risk of LBW in babies born to women who received 3 or more doses of IPTp-SP.

A major strength of this study is that unlike other studies that compared only $\geq 3$ doses of IPTp-SP to 2 doses, this study assessed the additional benefits or otherwise of receiving $\geq 4$ doses of IPTp-SP. This is one of very few studies ${ }^{30}{ }^{31}$ to evaluate the impact of the new WHO IPTp-SP policy and it was conducted in different ecological zones of Ghana with varied malaria transmission intensities, thereby producing generalisable results. A limitation of this study is that the study team was not directly involved in the administration of SP at the ANC 


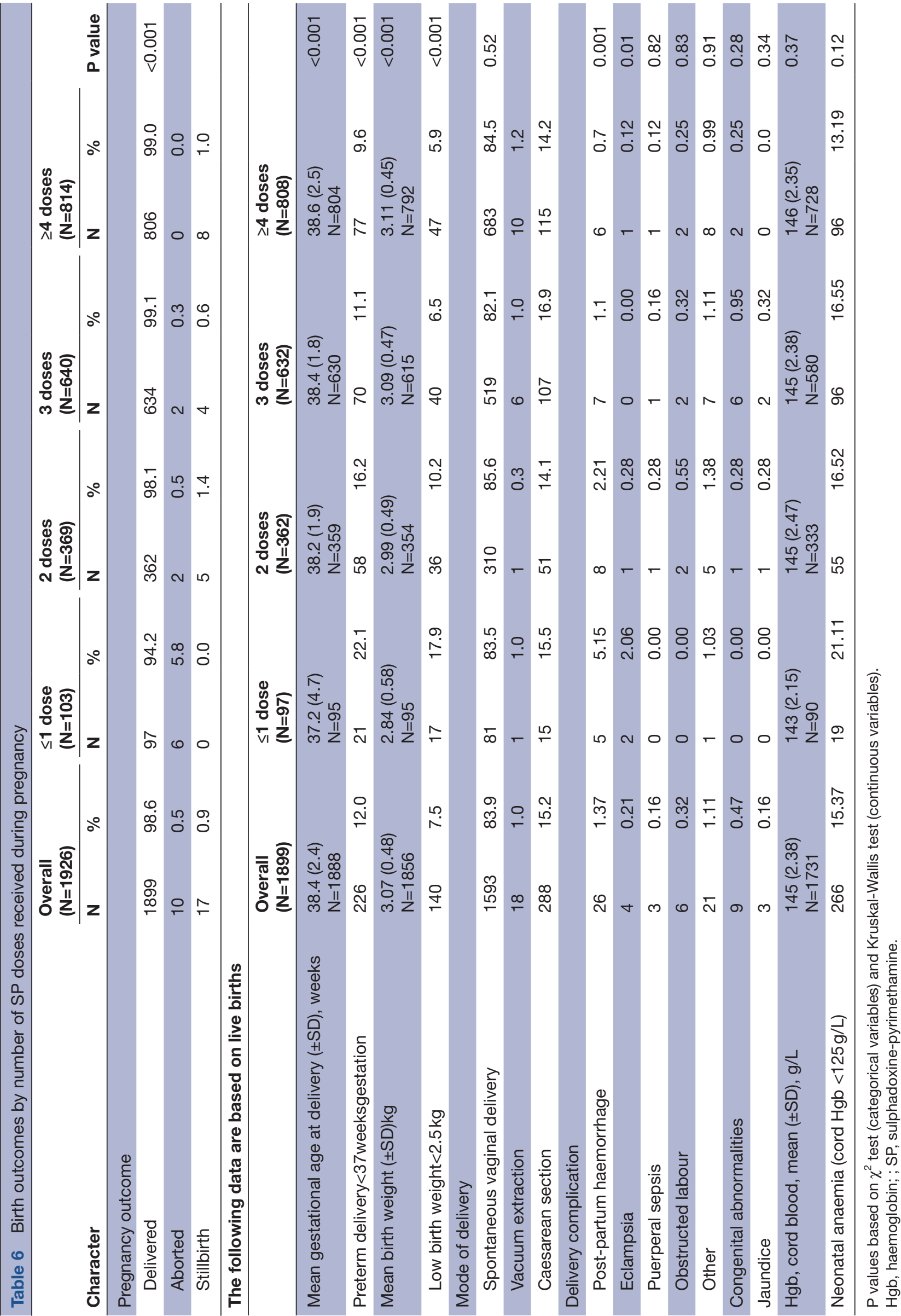

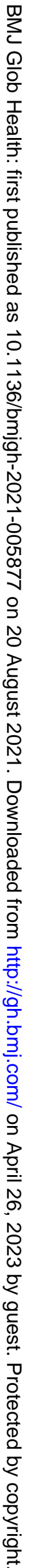




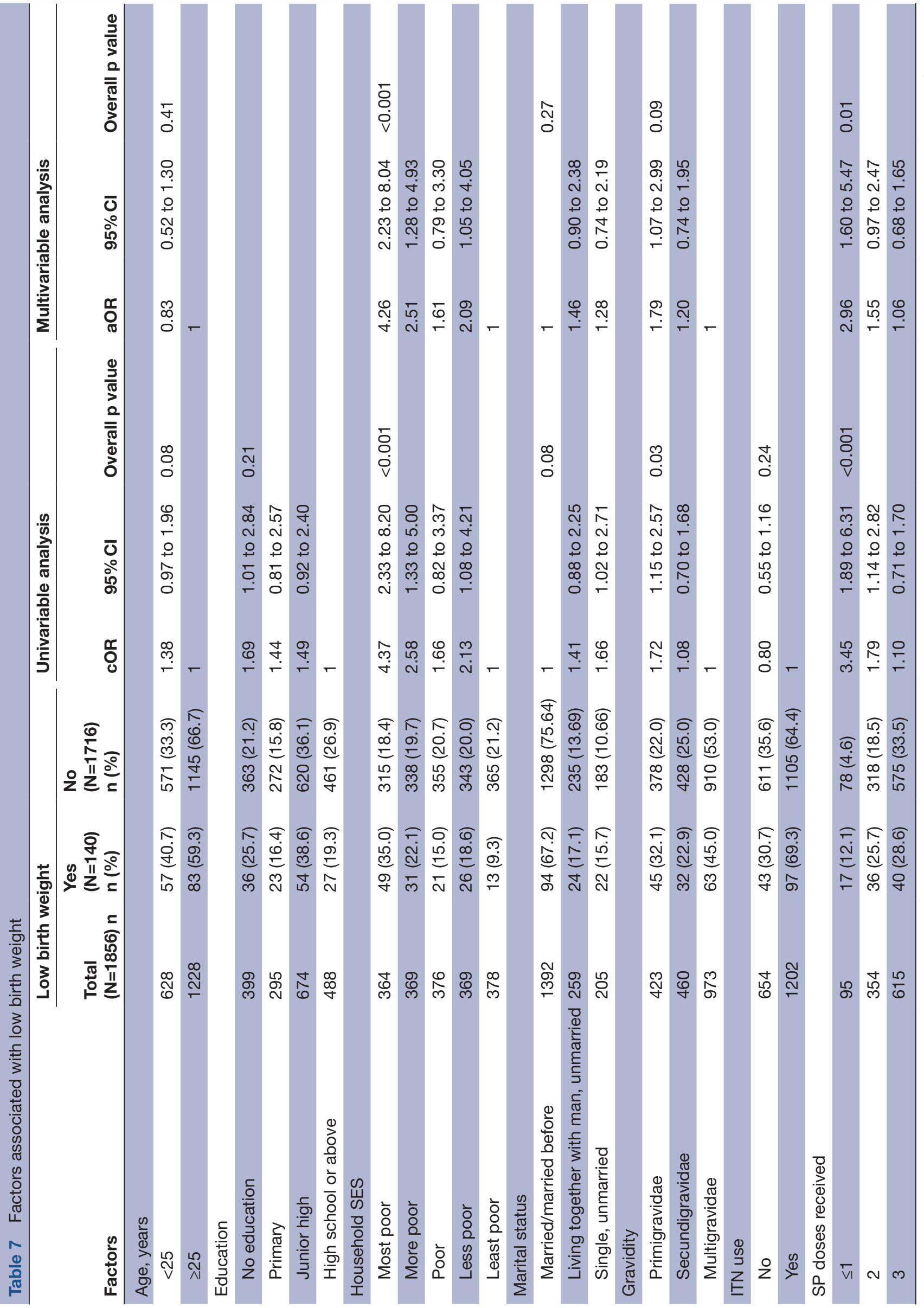




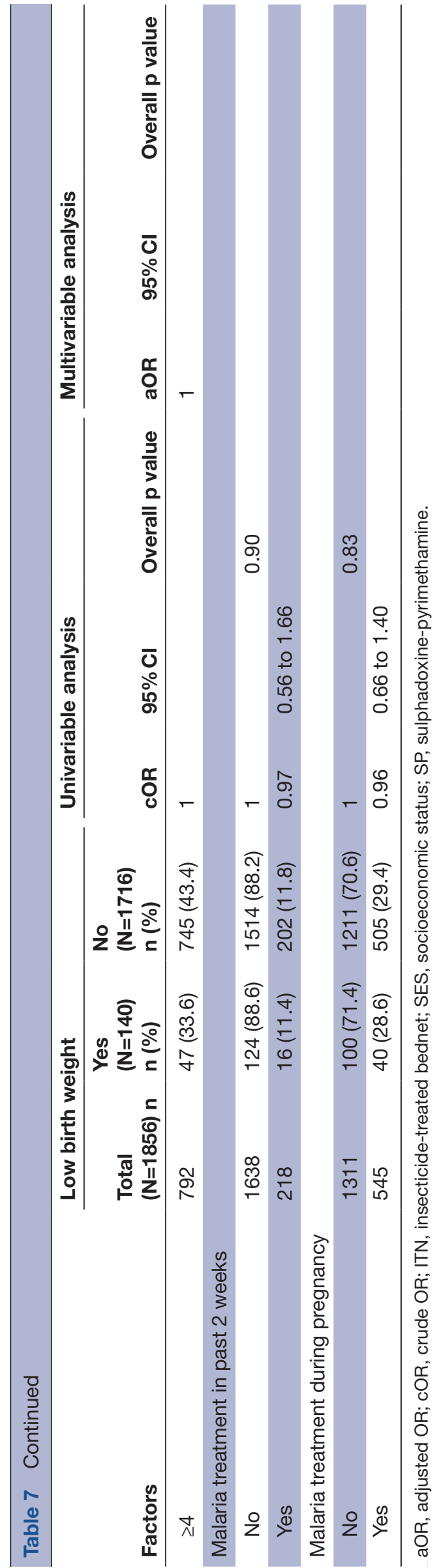

clinics, and consequently the number of doses of SP received was based on the number recorded in the ANC booklets of the pregnant women at the time of delivery. However, ANC nurses, who had previously been trained by the Ghana Health Service on administration of SP at the clinics, were given refresher training by the study team to ensure SP administration was directly observed at the clinic and accurately recorded in the women's booklets. A second limitation is that the possible impact of higher numbers of ANC visits on pregnancy outcomes could not be considered during the analysis. Previous studies have, however, shown that women with higher numbers of ANC visits have better pregnancy outcomes. ${ }^{32} 33$ Third, there is also the possibility that there were some important confounders associated with the number of doses given that could not be detected and corrected for in the multiple regression analysis. Fourth, another important limitation in this study is the lack of randomisation. With the commencement of the revised WHO policy on IPTpSP, it would have been unethical to assign participants to number of doses below the recommended number. This poses a risk for selection bias for which controlling was not possible. Another limitation was that multiple comparisons were done, so some statistically significant differences could have been found by chance. However, in general, the results were very consistent. Finally, even though no serious safety concerns were associated with monthly IPTp-SP dosing, this study was not powered to detect differences in safety indicators such as jaundice and congenital malformations, and measurement of serum bilirubin could have provided a more accurate estimates than clinical jaundice. Bilirubin could, however, not be measured due to logistical constrains.

This study did not show significant additional benefits of receiving $\geq 4$ doses compared with 3 doses IPTp-SP during pregnancy. Receiving 3 or more doses, however, showed a reduction in the risk of overall PM infection compared with 2 doses. The WHO policy of monthly administration of IPTp-SP to pregnant women in the second and third trimesters remains a more practical way of ensuring adequate doses are received during delivery, instead of restricting the number of doses to a maximum of three.

\section{Author affiliations}

${ }^{1}$ Department of Disease Control, London School of Hygiene and Tropical Medicine, Faculty of Infectious and Tropical Diseases, London, UK

${ }^{2}$ Kintampo Health Research Centre, Research and Development Division, Ghana Health Service, Kintampo, Ghana

${ }^{3}$ National Malaria Control Programme, Ghana Health Service, Accra, Ghana

${ }^{4}$ Department of Pathology, University of Ghana College of Health Sciences, Accra, Ghana

${ }^{5}$ Navrongo Health Research Centre, Research and Development Division, Ghana Health Service, Accra, Ghana

${ }^{6}$ Ghana Health Service Research and Development Division, Accra, Ghana ${ }^{7}$ Dodowa Health Research Centre, Research and Development Division, Ghana Health Service, Dodowa, Ghana

${ }^{8}$ Institute of Health Research, University of Health and Allied Sciences, Ho, Ghana

Acknowledgements The authors are grateful to the study participants, community members, hospital staff, Municipal and District Directors of Health Services for Kintampo North and South, Kassena-Nankana East and West, and 
Shai-0sudoku districts, and management and staff of the Kintampo, Navrongo and Dodowa Health Research Centres.

Contributors KPA, KM and DKD conceived the study. SO-A, NYP, DC, BG, AO and JW discussed and agreed on the study. DKD designed the laboratory studies. DKD and DA coordinated the study. DKD and RG performed the laboratory analysis. MT and DKD managed and cleaned data for analysis. FBO and DKD performed statistical analysis of the data. DKD wrote the first draft of the manuscript. All authors contributed to the interpretation of the results and revision of the manuscript. All authors read and approved the final manuscript.

Funding This study was funded by Global Fund/National Malaria Control Programme of Ghana and the Kintampo Health Research Centre.

Competing interests None declared.

\section{Patient consent for publication Not required.}

Ethics approval The study was approved by the ethics committees of the Kintampo Health Research Centre (KHRCIEC/2017-9) Ghana Health Service (GHSERC 02/07/2016) and the London School of Hygiene and Tropical Medicine (LSHTM Ethics Ref: 12336). Informed consent was obtained from all participants prior to enrolment into the study.

Provenance and peer review Not commissioned; externally peer reviewed.

Data availability statement Data are available upon reasonable request

Supplemental material This content has been supplied by the author(s). It has not been vetted by BMJ Publishing Group Limited (BMJ) and may not have been peer-reviewed. Any opinions or recommendations discussed are solely those of the author(s) and are not endorsed by BMJ. BMJ disclaims all liability and responsibility arising from any reliance placed on the content. Where the content includes any translated material, BMJ does not warrant the accuracy and reliability of the translations (including but not limited to local regulations, clinical guidelines, terminology, drug names and drug dosages), and is not responsible for any error and/or omissions arising from translation and adaptation or otherwise.

Open access This is an open access article distributed in accordance with the Creative Commons Attribution Non Commercial (CC BY-NC 4.0) license, which permits others to distribute, remix, adapt, build upon this work non-commercially, and license their derivative works on different terms, provided the original work is properly cited, appropriate credit is given, any changes made indicated, and the use is non-commercial. See: http://creativecommons.org/licenses/by-nc/4.0/.

\section{ORCID iD}

David Kwame Dosoo http://orcid.org/0000-0001-5152-462X

\section{REFERENCES}

1 Mlugu EM, Minzi O, Asghar M, et al. Effectiveness of SulfadoxinePyrimethamine for intermittent preventive treatment of malaria and adverse birth outcomes in pregnant women. Pathogens 2020;9:207.

2 Anchang-Kimbi JK, Kalaji LN, Mbacham HF, et al. Coverage and effectiveness of intermittent preventive treatment in pregnancy with sulfadoxine-pyrimethamine (IPTp-SP) on adverse pregnancy outcomes in the Mount Cameroon area, South West Cameroon. Malar J 2020;19:1-12.

3 Walker PGT, Floyd J, Ter Kuile F, et al. Estimated impact on birth weight of scaling up intermittent preventive treatment of malaria in pregnancy given sulphadoxine-pyrimethamine resistance in Africa: a mathematical model. PLoS Med 2017;14:e1002243.

4 World Health Organization. World malaria report 2019. Geneva: World Health Organization, 2019.

5 World Health Organization. A strategic framework for malaria prevention and control during pregnancy in the African region. A strategic framework for malaria prevention and control during pregnancy in the African region 2004.

6 Karunajeewa HA, Salman S, Mueller I, et al. Pharmacokinetic properties of sulfadoxine-pyrimethamine in pregnant women. Antimicrob Agents Chemother 2009;53:4368-76.

7 White NJ. Intermittent presumptive treatment for malaria. PLoS Med 2005;2:e3.

8 PMI. Ghana malaria operational plan FY 2014: president's malaria initiative 2014

9 Kayentao K, Garner P, van Eijk AM, et al. Intermittent preventive therapy for malaria during pregnancy using 2 vs 3 or more doses of sulfadoxine-pyrimethamine and risk of low birth weight in Africa: systematic review and meta-analysis. JAMA 2013;309:594-604.

10 Henry M, Florey L, Youll S, et al. An analysis of country adoption and implementation of the $2012 \mathrm{WHO}$ recommendations for intermittent preventive treatment for pregnant women in sub-Saharan Africa. Malar J 2018:17:364

11 Afari EA, Appawu M, Dunyo S, et al. Malaria infection, morbidity and transmission in two ecological zones southern Ghana. Afr J Health Sci 1995;2:312-5.

12 Owusu-Agyei S, Asante KP, Adjuik M, et al. Epidemiology of malaria in the forest-savanna transitional zone of Ghana. Malar J 2009;8:1-10.

13 Kasasa S, Asoala V, Gosoniu L, et al. Spatio-Temporal malaria transmission patterns in Navrongo demographic surveillance site, Northern Ghana. Malar J 2013;12:63-10.

14 Asante KP, Owusu-Agyei S, Cairns M, et al. Placental malaria and the risk of malaria in infants in a high malaria transmission area in Ghana: a prospective cohort study. J Infect Dis 2013;208:1504-13.

15 Dosoo DK, Chandramohan D, Atibilla D, et al. Epidemiology of malaria among pregnant women during their first antenatal clinic visit in the middle belt of Ghana: a cross sectional study. Malar $J$ 2020;19:1-12.

16 Lee ACC, Whelan R, Bably NN, et al. Prediction of gestational age with symphysis-fundal height and estimated uterine volume in a pregnancy cohort in Sylhet, Bangladesh. BMJ Open 2020;10:e034942.

17 Swysen C, Vekemans J, Bruls M, et al. Development of standardized laboratory methods and quality processes for a phase III study of the RTS, S/AS01 candidate malaria vaccine. Malar J 2011;10:223

18 World Health Organization. Basic malaria microscopy: part I. Learner's guide. Geneva: World Health Organization, 2010.

19 Asante KP, Owusu-Agyei S, Cairns M, et al. Placental malaria and the risk of malaria in infants in a high malaria transmission area in Ghana: a prospective cohort study. J Infect Dis 2013;208:1504-13.

20 Rogerson SJ, Pollina E, Getachew A, et al. Placental monocyte infiltrates in response to Plasmodium falciparum malaria infection and their association with adverse pregnancy outcomes. Am J Trop Med Hyg 2003;68:115-9.

21 Ismail MR, Ordi J, Menendez C, et al. Placental pathology in malaria: a histological, immunohistochemical, and quantitative study. Hum Pathol 2000;31:85-93.

22 World Health Organization. Haemoglobin concentrations for the diagnosis of anaemia and assessment of severity. Geneva: World Health Organization, 2011

23 Laar AK, Grant FE, Addo Y, et al. Predictors of fetal anemia and cord blood malaria parasitemia among newborns of HIV-positive mothers. BMC Res Notes 2013;6:350.

24 Firth D. Bias reduction of maximum likelihood estimates. Biometrika 1993;80:27-38

25 Gill CJ, Macleod WB, Mwanakasale V, et al. Inferiority of singledose sulfadoxine-pyrimethamine intermittent preventive therapy for malaria during pregnancy among HIV-positive Zambian women. $J$ Infect Dis 2007:196:1577-84.

26 Hamer DH, Mwanakasale V, Macleod WB, et al. Two-Dose versus monthly intermittent preventive treatment of malaria with sulfadoxine-pyrimethamine in HIV-seropositive pregnant Zambian women. J Infect Dis 2007;196:1585-94.

27 World Health Organization. WHO policy brief for the implementation of intermittent preventive treatment of malaria in pregnancy using sulfadoxine-pyrimethamine (IPTp-SP). Geneva: World Health Organization, 2013.

28 Tagbor H, Cairns M, Bojang K, et al. A non-inferiority, individually randomized trial of intermittent screening and treatment versus intermittent preventive treatment in the control of malaria in pregnancy. PLoS One 2015;10:e0132247.

29 Anto F, Agongo IH, Asoala V, et al. Intermittent preventive treatment of malaria in pregnancy: assessment of the sulfadoxinepyrimethamine three-dose policy on birth outcomes in rural Northern Ghana. J Trop Med 2019;2019:1-10.

30 Quakyi I, Tornyigah B, Houze P, et al. High uptake of intermittent preventive treatment of malaria in pregnancy is associated with improved birth weight among pregnant women in Ghana. Sci Rep 2019;9:1-8.

31 Agyeman YN, Newton SK, Annor RB, et al. The effectiveness of the revised intermittent preventive treatment with sulphadoxine pyrimethamine (IPTp-SP) in the prevention of malaria among pregnant women in northern Ghana. J Trop Med 2020;2020:1-9.

32 Appiah PK, Bukari M, Yiri-Erong SN, et al. Antenatal care attendance and factors influenced birth weight of babies born between June 2017 and may 2018 in the WA East district, Ghana. Int J Reprod Med 2020;2020:1-10.

33 Manjavidze T, Rylander C, Skjeldestad FE, et al. The impact of antenatal care utilization on admissions to neonatal intensive care units and perinatal mortality in Georgia. PLoS One 2020;15:e0242991. 